\title{
Possibility Based Modal Semantics for Graded Modifiers
}

\author{
Jorma K. Mattila \\ Lappeenranta University of Technology, \\ Department of Mathematics and Physics \\ P.O. Box 20, FIN-53851 Lappeenranta, Finland \\ jorma.mattila@lut.fi
}

\begin{abstract}
A brief introduction to basic modifiers is given. Any modifier with its dual and the corresponding negation form a DeMorgan triple similar to that of t-norms, t-conorms, and negation. The lattice structure of the unit interval with the usual partial order is similar to that of the set of all membership functions. This structure has a certain connection to implication, by means of the subsethood of fuzzy sets, and it is possible to create a similar expression for modifiers as the axiom of reflexivity is in modal logic. Also, other connections to modal logics can be found. This motivates to develop a formal semantics to modifier logic by means of that of modal logic. Actually, this kind of logic is so-called metalogic concerning either true or false statements about properties of modifiers. This version is based on graded possibility operations. Hence, semantic tools for weakening modifiers are derived. The corresponding things for substantiating modifiers are constructed by means of duality. Finally, some outlines for modifier systems are considered.
\end{abstract}

Keywords: Modality, Modifier, metalogic, Semantics of Modal Logic, Semantics of Modifier Logic, Modifier system.

\section{Basic Modifiers}

So-called Basic modifiers are modifiers having a certain effect from two possible properties. A basic modifier either substantiate or weaken the concept being its argument. For example, if the argument of a substantiating modifier is (as usually) a membership function, say $\mu$, then the modifier restricts $\mu$ in similar way at every point $x$ in the domain of the function. What this means, can be seen in Definition 1 below. We consider basic modifiers by giving the definitions and results as a short introduction to modifiers. We consider modifiers as operations in the set of membership functions

$$
\mathbb{I}^{X}=\{\mu \mid \mu: X \longrightarrow \mathbb{I}\}
$$

where $\mathbb{I}$ is the lattice $([0,1], \leq)$.

P. Melin et al. (Eds.): IFSA 2007, LNAI 4529, pp. 220230 2007.

(C) Springer-Verlag Berlin Heidelberg 2007 
Definition 1 (Modifier). We say that a mapping $M: \mathbb{I}^{X} \rightarrow \mathbb{I}^{X}$ is

(i) a substantiating modifier if for any fuzzy set $\mu \in \mathbb{I}^{X}$,

$$
\forall x \in X, \quad M(\mu(x)) \leq \mu(x),
$$

(ii) a weakening modifier if for any $\mu \in \mathbb{I}^{X}$,

$$
\forall x \in X, \quad \mu(x) \leq M(\mu(x)),
$$

(iii) an identity modifier if for any $\mu \in \mathbb{I}^{X}$,

$$
\forall x \in X, \quad M(\mu(x))=\mu(x) .
$$

Identity modifiers are identity mappings on $\mathbb{I}^{X}$. They are sometimes needed as links between substantiating and weakening modifiers in some logical structures involving modifier symbols.

Because a modifier $M$ is a map from $\mathbb{I}^{X}$ to $\mathbb{I}^{X}$, it has the properties of membership functions. A modifier $M$ is associated with a fuzzy set $\mu$ by means of composition of $M$ and $\mu$ as follows. For any $x \in X$,

$$
(M \circ \mu)(x)=M(\mu(x)) .
$$

A given modifier we can associate with the dual modifier according to the following

Definition 2 (Dual Modifier). Let $M$ and $M^{*}$ be modifiers. We say that $M^{*}$ is the dual modifier associated with $M$, if for any fuzzy set $\mu \in \mathbb{I}^{X}$,

$$
\forall x \in X, \quad M^{*}(\mu(x))=\eta(M(\eta(\mu(x)))),
$$

where $\eta$ is a strong negation.

Proposition 1. If $M$ is a substantiating modifier then its dual $M^{*}$ is a weakening modifier and vice versa.

Proof. (See also 13.) Suppose $\mu \in \mathbb{I}^{X}$, and $M$ is a substantiating modifier. Thus $\forall x \in X, M(\mu(x)) \leq \mu(x)$. We have to show that $\forall x \in X, \mu(x)=M^{*}(\mu(x))$. Let $n$ be a strong negation function. Thus $\forall x \in X, M^{*}((x))=\eta(M(\eta(\mu(x))))$. Clearly

$$
M(\eta(\mu(x))) \leq \eta(\mu(x))
$$

by Def 1 From this it follows by the properties of membership functions that

$$
\forall x \in X, \eta(M(\eta(\mu(x)))) \geq \eta(\eta(\mu(x)))=\mu(x),
$$

i.e.,

$$
M^{*}(\mu(x)) \geq \mu(x) .
$$

Conversely, the result follows in the similar way. 
The condition

$$
\forall x \in X, \quad M^{*}(\mu(x))=\eta(M(\eta(\mu(x))))
$$

in the previous proof says that the operators $M, M^{*}$, and $\eta$ satisfy DeMorgan's law. Thus dual pairs of modifiers with strong negation form classes called DeMorgan triples of operators ([2]). Originally, a DeMorgan triple consists of a t-norm, corresponding t-conorm, and negation, but the triples involving any modifier, its dual, and negation have the same structure by Definitions 1, 2, and Proposition 1 .

We denote $\alpha$-level set of a fuzzy set $\mu$, as usually,

$$
\mu_{\alpha}=\{x \in X \mid \mu(x) \geq \alpha, \alpha \in \mathbb{I}\}
$$

Thus the $\alpha$-level set of $M(\mu)$ is

$$
(M \circ \mu)_{\alpha}=\{x \in X \mid M(\mu(x)) \geq \alpha, \alpha \in \mathbb{I}\} .
$$

It is easy to see that modifiers have following properties. Suppose $M$ is a substantiating modifier. Then we have

$$
\begin{aligned}
M\left(\mathbf{0}_{X}\right) & =\mathbf{0}_{X}, \\
M^{*}\left(\mathbf{1}_{X}\right) & =\mathbf{1}_{X}, \\
\left(M^{*}\right)^{*}(\mu(x)) & =M(\mu(x)),
\end{aligned}
$$

where $\mathbf{0}_{\mathbf{X}}$ and $\mathbf{1}_{X}$ are the constant functions $\mathbf{0}_{X}(x)=0$ and $\mathbf{1}_{X}(x)=1$ for all $x \in X$.

The lattice structure of $\mathbb{I}$ gives the natural ordering to a set of modifiers, similar to that of any set of membership functions. This is an implication of the well known fact that the lattice properties of $\mathbb{I}$ can be embedded pointwise to the lattice $\mathbb{I}^{X}$. Hence, because $\mathbb{I}$ is a distributive and complete lattice, so is $\mathbb{I}^{X}$, too. The partial ordering relation " $\leq$ " involves the principle of implication. This means that if $M$ is a substantiating modifier then for any $x \in X$ and $\mu \in \mathbb{I}^{X}$, $M(\mu(x)) \leq \mu(x)$, by Definition 1. In fuzzy set theory, this means that a fuzzy set $M(\mu)$ is a subset of the fuzzy set $\mu$ (cf. Zadeh [16]). Hence, if we think that $M$ is the linguistic label of the formal modifier $M$, i.e., a suitable hedge corresponding to the formal $M$, and $\mu$ is the linguistic interpretation of the formal fuzzy set $\mu$, then the statement

$$
M(\mu) \rightarrow \mu
$$

is an implication being true in any situation where for any $x \in X$ and $\mu \in \mathbb{I}^{X}$, $M(\mu(x)) \leq \mu(x)$. This means that the statement

$$
\mu \rightarrow M^{*}(\mu)
$$

is true in some possible worlds. In the same way, we can consider that the statement $\mu \rightarrow M^{*}(\mu)$ is true in the same possible worlds, by Definition 2 and 
Proposition [1. If we consider $M$ to be a necessity operator and $M^{*}$ a possibility operator, then the formula (13) represents the axiom of reflexivity in systems where the primitive modal operator is necessity and (14) in modal systems where the primitive modal operator is possibility.

When we consider modifiers as logical operators, we have to notice that they are not truth-functional. This means that the truth values of modified statements do not depend only of the truth value distributions of the primitive symbols in one world, but also of the accessibility to other possible worlds. This is an essential presupposition for the possibility to interpret modifiers as modalities. We will see this in the considerations below.

\section{Modifier Language}

First, consider some fundamental things from history and some motivating things. Lemmon [8], p. 20 - 21, describes Leibniz' basic ideas for motivating the idea of "classical modal logics". He says: "Leibniz' suggestion now becomes: a sentence is necessarily true (in this world) iff that sentence is true in all worlds alternative to this world." See Lemmon [8], p. 20 to check the detailed analysis about what the concept "alternative to this world" means. Lemmon [8], p. 21, continues: "Actually, in many connections it is intuitively simpler to think of world $t$ as accessible from world $u$ rather than alternative to $u$. This at least has the merit of avoiding the temptation to suppose that alternativeness is a symmetric relation between worlds - that if $t$ is alternative to $u$, then $u$ must be alternative to $t$. Indeed, we shall not assume that each world is accessible from itself, or even that to each world there is at least one accessible world: there may be accessibility-isolated worlds. We shall find that to many such assumptions about the accessibility relation between worlds there correspond distinctive modal sentences which come out valid precisely because we have made those assumptions. If necessity means truth in all accessible worlds, then possibility will mean truth in some accessible world. Thus our remarks about the vagueness of the notion of necessity, and the various more precise accounts of it, may be repeated mutantis mutandis for the notion of possibility."

Traditional modal operators are actually modifiers that substantiate or weaken statements or their parts being arguments of the operators. For example, the concepts possible and necessary make indefinite (i.e. weaken) and make necessary (i.e. substantiate) expressions associated with them, respectively. Thus modal logics can be viewed to be logics of modifiers. We define modifiers formally by means of relational structures, or Kripke frames, when they can be interpreted in different ways. We restrict our consideration only to the cases where modifiers are unary operators.

Propositional modifier language $\mathcal{L}_{\text {Mod }}$ is a language of graded modalities, that can be generally defined by giving its alphabet and rules of formation of formulas as follows. 
Definition 3. The alphabet of a language $\mathcal{L}_{\text {Mod }}$ consist of:

(i) propositional letters $p, q, \ldots(\in \operatorname{Prop})$;

(ii) binary truth-functional connectives;

(iii) zero-placed operator $\perp$ (fixes the value false);

(iv) non-truth-functional sentence operators $\boldsymbol{F}_{1}, \boldsymbol{F}_{2}, \ldots(\in \mathbb{O})$.

Definition 4. Well-formed formulas (wff's or formulas, for short) of a language $\mathcal{L}_{M o d}$ are as follows:

(i) propositional letters $p, q, \ldots(\in$ Prop $)$ are formulas;

(ii) $\perp$ is a formula;

(iii) if $\alpha$ and $\beta$ are formulas, and $\downarrow$ is any binary truth-functional connective, then $\alpha \beta$ is a formula;

(iv) if $\alpha$ is a formula, and $\boldsymbol{F}_{i} \in \mathbb{O}$ then $\boldsymbol{F}_{i}(\alpha)$ is a formula $(i=1,2, \ldots)$.

Formulas of the form $\alpha \rightarrow \perp$ are usually abbreviated by $\neg \alpha$.

\section{Graded Possibility Based Modal Semantics}

We consider a relation system

$$
\mathbf{R}=\bigcup_{i=0}^{k} \mathbf{R}_{i}
$$

of binary subrelations of a given binary relation $\mathbf{R}$. It is the central part of relational structures we are considering when constructing frames and models for logics of graded modifiers. The relations may have any properties as relations usually have. For example, they are reflexive, symmetric, transitive etc. The only thing we presuppose is that the relations are at least reflexive. Then we can generate systems being similar to aletic modal systems.

Definition 5. frame, or relational structure, is an ordered queue

$$
\mathcal{F}=\left\langle W, \mathbf{R}_{0}, \mathbf{R}_{1}, \mathbf{R}_{2}, \ldots, \mathbf{R}_{k}\right\rangle
$$

where $W \neq \emptyset$ is a set and $\mathbf{R}_{i}$ is a binary relation, $i=1, \ldots, k$. Elements $w$ of $W$ are points, states, or possible worlds.

A model corresponding to the frame $\mathcal{F}$ is an ordered queue

$$
\mathcal{M}=\left\langle W, \mathbf{R}_{0}, \mathbf{R}_{1}, \mathbf{R}_{2}, \ldots, \mathbf{R}_{k}, V\right\rangle
$$

where $V:$ Prop $\rightarrow \mathcal{P}(W)$ is a valuation.

For every world $w \in W, V$ determines a set of those propositional letters, which are true in $w$, namely the set

$$
P_{w}=\{p \mid w \in V(p)\} .
$$


The number of relations $k$ may be finite or denumerably infinite.

Every possible world $w$ is a maximal consistent set of formulas, i.e. $\alpha \rightarrow \beta \in w$ iff $\alpha \notin w$ or $\beta \in w$, and $\alpha \in w$ iff $\alpha \rightarrow \perp \notin w$, i.e. $\neg \alpha \notin w$. Thus a set of worlds $W$ is a set of maximal consistent sets of formulas. We can now define modifiers by means of relations $\mathbf{R}_{i}(i=0,1,2, \ldots, k)$ of a Kripke frame as follows.

Definition 6. Let $\mathcal{F}=\left\langle W, \mathbf{R}_{0}, \mathbf{R}_{1}, \mathbf{R}_{2}, \ldots, \mathbf{R}_{k}\right\rangle$ be a frame, $\alpha$ a formula of $\mathcal{L}_{\text {Mod }}$, and $w, t \in W$. Then for every value of the index $i=0,1,2, \ldots, k, \boldsymbol{H}_{i}$ is a possibility operator, (i.e., a weakening modifier) iff the condition

$$
w \mathbf{R}_{i} t \Leftrightarrow\left\{\boldsymbol{H}_{i}(\alpha) \mid \alpha \in t\right\} \subseteq w
$$

holds, i.e. $w \mathbf{R}_{i} t$ holds iff for every formula $\alpha \in t$, for which $\boldsymbol{H}_{i}(\alpha) \in w$. Then we say that $\boldsymbol{H}_{i}$ is a possibility operator determined by $\mathbf{R}_{i}$. A set of relations $\left\{\mathbf{R}_{0}, \mathbf{R}_{1}, \mathbf{R}_{2}, \ldots, \mathbf{R}_{k}\right\}$ is indicated by $S_{\mathbf{R}}$.

In the sequel, we use the name "modifier" for the modal operators.

Definition 7. The dual $\boldsymbol{H}_{i}^{*}$ of a modifier $\boldsymbol{H}_{i}$ is defined by the condition

$$
\boldsymbol{H}_{i}^{*}(\alpha) \equiv \neg \boldsymbol{H}_{i}(\neg \alpha)
$$

(三 is a symbol of metalanguage presenting equivalence relation between two formulas.)

A set of duals of modifiers defined in Def. [6] is indicated by $\mathbb{O}^{*}$, i.e.

$$
\mathbb{O}^{*}=\left\{\boldsymbol{H}_{0}^{*}, \boldsymbol{H}_{1}^{*}, \ldots, \boldsymbol{H}_{k}^{*}\right\} .
$$

Elements of the set (21) are weakening modifiers, and we say that $\boldsymbol{H}_{i}^{*}$ is a substantiating modifier determined by $\mathbf{R}_{i}$.

Proposition 2. Let $\mathbf{R}_{i} \in S_{\mathbf{R}}, \boldsymbol{H}_{i} \in \mathbb{O},(i=0,1, \ldots, k)$, and $\alpha$ a formula of $\mathcal{L}_{\text {Mod }}$. Then the formula

$$
w \mathbf{R}_{i} t \Leftrightarrow\left\{\alpha \mid \boldsymbol{F}_{i}(\alpha) \in w\right\} \subseteq t
$$

holds.

Proof. Suppose $w \mathbf{R}_{i} t, \boldsymbol{F}_{i}(\alpha) \in w$, and $\alpha \notin t$. Then $\neg \alpha \in t$, because $t$ is maximal consistent, and further, $\boldsymbol{H}_{i}(\neg \alpha) \in w$ by (19). From this the condition $\neg \boldsymbol{F}_{i}(\alpha) \in$ $w$ follows by Def. 7. But this is in contradiction to the supposition, because $w$ is maximally consistent. Thus $w \mathbf{R}_{i} t \Rightarrow\left\{\alpha \mid \boldsymbol{F}_{i}(\alpha) \in w\right\} \subseteq t$.

In the converse case, suppose $\boldsymbol{F}_{i}(\alpha) \in w$ for all formulas $\alpha \in t$. From this it follows that $\neg \boldsymbol{F}_{i}(\alpha) \notin w$ by maximal consistency of $w$, i.e. $\boldsymbol{H}_{i}(\neg \alpha) \notin w$ by Def. 7 , and also $\neg \alpha \notin t$ by maximal consistency of $t$. Thus $\boldsymbol{H}_{i}(\neg \alpha) \notin w \Rightarrow$ $\neg \alpha \in t$, from which the implication $\alpha \in t \Rightarrow \boldsymbol{H}_{i}(\alpha) \in w$ by contraposition. Thus $\left\{\boldsymbol{H}_{i}(\alpha) \mid \alpha \in t\right\} \subseteq w$, and thus $w \mathbf{R}_{i} t$ by (19). This completes the proof. 
The truth status of a formula in a given world $w$ follows the principle, according to which $w$ is closed under connectives.

Definition 8. A truth value of a formula $\alpha$ of $\mathcal{L}_{M o d}$ in a given world $w$ of a model $\mathcal{M}=\left\langle W, \mathbf{R}_{0}, \mathbf{R}_{1}, \mathbf{R}_{2} \ldots, \mathbf{R}_{k}, V\right\rangle$ (denote $\models_{w}^{\mathcal{M}} \alpha$ ) is defined recursively as follows:

(i) If $p \in$ Prop then $\models_{w}^{\mathcal{M}} p$ iff $V(p)$.

If $\alpha$ and $\beta$ are formulas and $\boldsymbol{F}_{i} \in \mathbb{O}(i=0,1,2, \ldots, k)$ then

(ii) $\models{ }_{w}^{\mathcal{M}} \neg \alpha$ iff $\not{ }_{w}^{\mathcal{M}} \alpha$;

(iii) $\models{ }_{w}^{\mathcal{M}} \alpha \rightarrow \beta$ iff $\forall_{w}^{\mathcal{M}} \alpha$, or $\models{ }_{w}^{\mathcal{M}} \beta$;

(iv) $\models{ }_{w}^{\mathcal{M}} \boldsymbol{F}_{i}(\alpha)$ iff for every world $t$, such that if $w \mathbf{R}_{i} t$ then $\models_{t}^{\mathcal{M}} \alpha$.

A truth condition for the dual $\boldsymbol{F}_{i}^{*}$ of the operator $\boldsymbol{F}_{i} \in \mathbb{O}(i=0,1,2, \ldots, k)$ can be derived by means of Def. 8 (iv). Let $\alpha$ be a formula of $\mathcal{L}_{M o d}$, then

$(\mathrm{v}) \models{ }_{w}^{\mathcal{M}} \boldsymbol{F}_{i}^{*}(\alpha)$ iff there exists $t \in W$, such that $w \mathbf{R}_{i} t$ and $\models_{t}^{\mathcal{M}} \alpha$.

A line of argument for the case (v) is analogous to that for $\diamond$ in Chellas' book [1] on page 7.

For every formula $\alpha$ in $\mathcal{L}_{M o d}$ and for every model $\mathcal{M}$ there exists a set

$$
\|\alpha\|^{\mathcal{M}}=\left\{w \in W \mid \models{ }_{w}^{\mathcal{M}} \alpha\right\} .
$$

This set is called a truth set of $\alpha$ in a model $\mathcal{M}$.

Suppose that a frame $\mathcal{F}=\left\langle W, \mathbf{R}_{0}, \mathbf{R}_{1}, \mathbf{R}_{2} \ldots, \mathbf{R}_{k}\right\rangle$ is given. We say that a formula $\alpha$ in $\mathcal{L}_{\text {Mod }}$ is valid in $\mathcal{F}$, denote $\models^{\mathcal{F}} \alpha$ iff $\models_{w}^{\mathcal{M}} \alpha$ holds in all models $\mathcal{M}$ of $\mathcal{F}$, and $w \in W$. A formula $\alpha$ is satisfiable in $\mathcal{F}$ iff $\models_{w}^{\mathcal{M}} \alpha$ holds in some models $\mathcal{M}$ of $\mathcal{F}$, and $w \in W$. A formula $\alpha$ is valid iff $\alpha$ is valin in every $\mathcal{F}$, and satisfiable iff $\alpha$ is satisfiable in some $\mathcal{F}$. Equivalently, we can say that $\alpha$ is valid iff $\models{ }_{w}^{\mathcal{M}} \alpha$ holds in all models $\mathcal{M}=\left\langle W, \mathbf{R}_{0}, \mathbf{R}_{1}, \mathbf{R}_{2} \ldots, \mathbf{R}_{k}, V\right\rangle$, and $w \in W$. It is also very often natural to use the concept "validity of a formula $\alpha$ in a model $\mathcal{M}$ ", denote $=^{\mathcal{M}} \alpha$. We say that $\alpha$ is valid in $\mathcal{M}$ iff $=_{w}^{\mathcal{M}} \alpha$ holds in all $w \in W$. Clearly, $\models^{\mathcal{F}} \alpha$ iff $\models^{\mathcal{M}} \alpha$ for every $\mathcal{M}$ in $\mathcal{F}$, and $\models \alpha$ iff $\models{ }^{\mathcal{M}} \alpha$ for every $\mathcal{M}$.

Definition 9. Semantic entailment $\alpha \models \beta$ of formulas $\alpha$ and $\beta$ in $\mathcal{L}_{M o d}$ is defined by the condition

$$
\alpha \models \beta \text { iff } \models \alpha \rightarrow \beta \text {. }
$$

Semantic entailment has a special property concerning modifier symbols. It can be proved that the following thing holds:

$$
\boldsymbol{H}(\alpha)=\beta \text { iff } \models \alpha \rightarrow \beta,
$$

$\left(\operatorname{Ent}_{W E A K}\right)$

where $\boldsymbol{H}$ is a weakening modifier symbol. The corresponding result for any substantiating modifier symbol is

$$
\alpha \models \boldsymbol{F}(\beta) \text { iff } \models \alpha \rightarrow \beta,
$$

$\left(\operatorname{Ent}_{S U B S T}\right)$

where $\boldsymbol{F}$ is any substantiating modifier symbol. 


\section{Towards Modifier Systems}

We consider here some preliminary things we need in creating modifier systems using the semantics considered in Sec. 3. We will have different systems by giving different properties to relations of frames $\mathcal{F}=\left\langle W, \mathbf{R}_{0}, \mathbf{R}_{1}, \mathbf{R}_{2} \ldots, \mathbf{R}_{k}\right\rangle$. Especially, we fix the relation $\mathbf{R}_{0}$ for a certain purpose. We give the property

$$
w \mathbf{R}_{0} t \Leftrightarrow w=t \text { for all } w, t \in W
$$

to it. In this case, the equivalence

$$
w \mathbf{R}_{0} t \Leftrightarrow\left\{\alpha \mid \boldsymbol{F}_{0}(\alpha) \in w\right\} \subseteq w \Leftrightarrow\left\{\boldsymbol{F}_{0}^{*}(\alpha) \mid \alpha \in t\right\} \subseteq w
$$

follows from the formulas (19) and (22). From this it follows that for such formulas $\alpha$ belonging to the set $\left\{\alpha \mid \boldsymbol{F}_{0}(\alpha) \in w\right\}$, the condition

$$
\alpha \in w \Leftrightarrow \boldsymbol{F}_{0}^{*}(\alpha) \in w
$$

holds, and thus $\|\alpha\|^{\mathcal{M}}=\left\|\boldsymbol{F}_{0}^{*}(\alpha)\right\|^{\mathcal{M}}$. From the corollary (v) of Def. 8 (vi) we have the condition

$$
\models{ }_{w}^{\mathcal{M}} \boldsymbol{F}_{0}^{*}(\alpha) \Leftrightarrow={ }_{w}^{\mathcal{M}} \alpha
$$

by (24), and this leads to the identity $\|\alpha\|^{\mathcal{M}}=\left\|\boldsymbol{F}_{0}^{*}(\alpha)\right\|^{\mathcal{M}}$. On the other hand, from Def. 8 (iv) the condition

$$
\models_{w}^{\mathcal{M}} \boldsymbol{F}_{0}(\alpha) \Leftrightarrow \models{ }_{w}^{\mathcal{M}} \alpha
$$

follows by (24). From this it follows that $\|\alpha\|^{\mathcal{M}}=\left\|\boldsymbol{F}_{0}(\alpha)\right\|^{\mathcal{M}}$. By means of these identities the result

$$
\left\|\boldsymbol{F}_{0}^{*}(\alpha)\right\|^{\mathcal{M}}=\left\|\boldsymbol{F}_{0}(\alpha)\right\|^{\mathcal{M}} .
$$

This result can be interpreted by the conclusion that the operator $\boldsymbol{F}_{0}$ is its own dual. It can be thought that the formulas $\alpha, \boldsymbol{F}_{0}(\alpha)$ and $\boldsymbol{F}_{0}^{*}(\alpha)$, whose truth sets are identical, express in each world exactly same states of affairs, and, in this point of view, they are equivalent. When we define the equivalence of two formulas in this way, then especially from the equivalence

$$
\alpha \equiv \boldsymbol{F}_{0}^{*}(\alpha)
$$

we get a result concerning the selfduality of $\boldsymbol{F}_{0}$ as follows:

$$
\begin{aligned}
\boldsymbol{F}_{0}(\alpha) & \stackrel{28}{\equiv} \boldsymbol{F}_{0}\left(\boldsymbol{F}_{0}^{*}(\alpha)\right) \equiv \boldsymbol{F}_{0}\left(\neg \boldsymbol{F}_{0}(\neg \alpha)\right) \equiv \neg \boldsymbol{F}_{0}^{*}\left(\boldsymbol{F}_{0}(\neg \alpha)\right) \\
& \stackrel{28}{\equiv} \neg \boldsymbol{F}_{0}(\neg \alpha) \equiv \boldsymbol{F}_{0}^{*}(\alpha) .
\end{aligned}
$$

From this it also follows that the sets in (25) are idetical, i.e.

$$
\left\{\alpha \mid \boldsymbol{F}_{0}(\alpha) \in w\right\}=\left\{\boldsymbol{F}_{0}^{*}(\alpha) \mid \alpha \in t\right\}
$$


Thus the operator $\boldsymbol{F}_{0}$ is an identity operator denoted often by $\Im$.

Next, we consider some additions to the formal semantics considered above. The purpose for this is to give some preliminary things for creating modifier systems, i.e. for choosing a set of axiom scheme and sets of inference rules. These additions concern mainly properties of relations of frames. All the systems use identity operator, and thus the relation $\mathbf{R}_{0}$ existing in the frames satisfies the condition (24) and determines an identity operator by means of considerations above basing on (19) and (22). The validity of used axioms has been proved in Mattila [11. Every possible world $w \in W$ mentioned in frames is closed under modus ponens, and thus modus ponens

$$
\alpha \rightarrow \beta, \alpha \vdash_{S} \beta
$$

appears as an inference rule in every systems. The subscript $S$ in the symbol $\vdash_{S}$ refers to the system under consideration. In addition to this, according to the basic semantics considered above the (semantical) rule of possibility is as follows. Let $\mathbf{H}_{i}$ be any weakening modifier operator, then

$$
\models \boldsymbol{H}_{i}(\alpha) \Rightarrow \models \alpha
$$

holds for all $\boldsymbol{H}_{i} \in \mathbb{O}^{*}$, and thus its syntactical counterpart

$$
\vdash_{S} \quad \boldsymbol{H}_{i}(\alpha) \Rightarrow \vdash_{S} \quad \alpha
$$

is used as a second inference rule. The duals of the rule of possibility $\left(\mathrm{RP}_{S E M}\right.$ and its syntactical counterpart $(\underline{\mathrm{RP}})$ are rule of substantiation

$$
\vDash \alpha \Rightarrow \models \boldsymbol{F}_{i}(\alpha)
$$

that holds for all $\boldsymbol{F}_{i} \in \mathbb{O}$, and its syntactical counterpart is

$$
\vdash_{S} \quad \alpha \Rightarrow \vdash_{S} \quad \boldsymbol{F}_{i}(\alpha)
$$

The rules $\left(\mathrm{RS}_{S E M}\right.$ and $(\underline{\mathrm{RS}})$ follow from the rules $\left(\mathrm{RP}_{S E M}\right.$ and $(\underline{\mathrm{RP}}$, respectively, by the principle of duality. The rule ( $\mathrm{RS}$ ) is a generalization of the rule of necessitation (RN) in usual modal logic.

\section{Concluding Remarks}

Modifier logics based on graded modalities are two-valued logics concerning statements about properties and relations of modifiers where the statements are either true or false. Hence, modifier logic can be considered to be so-called metalogic considering things in metalevel. Hence, metalogic of basic modifiers is not classical logic but logic of graded modal operators. This means that it is not truth-functional, because modifier operators in modifier logic are not truthfunctional.

From the definitions and results in Section 1 concerning modifiers, it seems to be possible to create an alternative formal semantics for aletic modal logic using 
the structure of modifiers. Some hints to this direction exist already in above considered things.

The set of relations

$$
S_{\mathbf{R}}=\left\{\mathbf{R}_{0}, \mathbf{R}_{1}, \mathbf{R}_{2}, \ldots, \mathbf{R}_{k}\right\}
$$

is naturally partially ordered by the ordering " $\subseteq$ ". If the ordering is linear, then we have a nested queue

$$
\mathbf{R}_{0} \subseteq \mathbf{R}_{1} \subseteq \mathbf{R}_{2} \subseteq \ldots \subseteq \mathbf{R}_{k}
$$

where it is possible that $\mathbf{R}_{k}=\mathbf{R}$, by 15 (or otherwise, $\mathbf{R}_{k} \subset \mathbf{R}$ ). If the condition (29) holds with $\mathbf{R}_{k}=\mathbf{R}$, then it is quite easy to create modifier systems where all the modifiers can be linearly ordered according to the strength of the modifier. Actually, the strength of the modifiers of a system can be defined by means of a nested relation system.

In any case, it is possible to create modifier systems with completeness results similar to graded systems $\mathbf{T}, \mathbf{S} 4$, or $\mathbf{S 5}$, if the relations are reflexive, reflexive and symmetric, or reflexive, symmetric and transitive, respectively. In the nested case, see for example [9] and [12].

Instead of possibility operations, it is also possible to create similar semantics for modifiers using necessity operators, i.e., substantiating modifiers. The basic starting point to that approach is the same as here (cf. [12]).

\section{References}

1. B. F. Chellas, Modal Logic: An Introduction, Cambridge University Press, Cambridge, 1980 .

2. J. Dombi, A fuzzy halmazok operátorainak szerkezete a többtényezós döntések szempontjából, Kandidátusi Értekezés, Hungarian Academy of Sciences, Szeged, 1993.

3. J. Dombi, Theoretical Concepts of Modifiers and Hedges, in: V.A. Niskanen, Jari Kortelainen (eds.), On the Edge of Fuzziness, Acta Universitatis Lappeenrantaensis 179, Lappeenranta, 2004.

4. B. Habson, P. Gärdenfors, A guide to intensional semantics, in: Modality, Morality and Other Problems of Sense and Nonsense: Essays Dedicated to Sören Halldén, CWK Gleerup Bokförlag, Lund, 1973.

5. G. E. Hughes, M. J. Cresswell, An Introduction to Modal Logic, Methuen and Co., reprint, 1985.

6. S. A. Kripke, Semantical considerations on modal logic, Acta Philosophica Fennica, Fasc. XVI, 1963.

7. G. Lakoff, Hedges: A study in meaning criteria and the logic of fuzzy concepts, The Journal of Philosophical Logic, 2, 1973.

8. E. J. Lemmon, An Introduction to Modal Logic, American Philosophical Quarterly, Monograph No. 11 (K. Segerberg (ed.)), Oxford, 1977.

9. J. K. Mattila, Modeling fuzziness by Kripke structures, in: T. Terano, M. Sugeno, M. Mukaidono, K. Shigemasu (eds.), Fuzzy Engineering toward Human Friendly Systems, Vol. 2, Proc. of IFES '91, Nov. 13 - 15, 1991, Yokohama, Japan. 
10. J. K. Mattila, On modifier logic, in: L. A. Zadeh, J. Kacprzyk (eds.), Fuzzy Logic for Management of Uncertainty, John Wiley \& Sons, Inc., New York, 1992.

11. J. K. Mattila, Completeness of propositional modifier logic, Research Report 55, Lappeenranta University of Technology, Department of Information Technology, 1995

12. J. K. Mattila, Modifier Logics Based on Graded Modalities, Journal of Advanced Computational Intelligence and Intelligent Informatics, Vol. 7 No. 2, 2003

13. J. K. Mattila, Modifiers Based on Some t-norms in Fuzzy Logic, Soft Computing, Springer-Verlag, DOI 10.1007/soo5oo-003-0323-x, 2003 (online), 2004 (paper).

14. J.K. Mattila, On Łukasiewicz Modifier Logic, Journal of Advanced Computational Intelligence and Intelligent Informatics, Vol. 9 No. 5, 2005

15. G. Resconi, G. J. Klir, U. H. St. Clair, Uncertainty and modal logic, Proceedings of Fifth International Fuzzy Systems Association World Congress'93, Vol. I, July 4 - 9, 1993, Seoul, Korea, 1993

16. L.A. Zadeh, Fuzzy Sets, Information and Control, 8, 1965. 